\title{
Amygdala Regulation of Nucleus Accumbens Dopamine Output is Governed by the Prefrontal Cortex
}

\author{
Mark E. Jackson and Bita Moghaddam \\ Department of Psychiatry, Yale University School of Medicine, West Haven, Connecticut 06516
}

A dynamic interaction between the prefrontal cortex (PFC), amygdala, and nucleus accumbens (NAc) may be fundamental to regulation of goal-directed behavior by affective and cognitive processes. This study demonstrates that a mechanism for this triadic relationship is an inhibitory control by prefrontal cortex on accumbal dopamine release during amygdala activation. In freely moving rats, microstimulation of basolateral amygdala at intensities that produced mild behavioral activation produced an expected rapid increase in glutamate efflux in the prefrontal cortex and the nucleus accumbens shell region of the ventral striatum. However, during the stimulation, dopamine release increased only in the prefrontal cortex, not in the nucleus accumbens. An increase in accumbal dopamine release was observed during the stimulation if glutamate activation in the prefrontal cortex was inhibited at either presynaptic or postsynaptic levels. Some behaviors expressed during the stimulation were intensified in animals in which prefrontal cortex glutamate activation was blocked. In addition, these animals continued to express stimulus-induced behaviors after the termination of stimulation, whereas normal poststimulus behaviors such as ambulation and grooming were not displayed as frequently. Considering that dopamine neurotransmission in the nucleus accumbens is thought to play an integral role in goaldirected motor behavior, these findings suggest that the prefrontal cortex influences the behavioral impact of amygdala activation via a concomitant active suppression of accumbal dopamine release. Absence of this cortical influence appears to result in an aberrant pattern of behavioral expression in response to amygdala activation, including behavioral perseveration after stimulus termination.

Key words: AMPA; striatum; ventral tegmental area; metabotropic glutamate receptors; schizophrenia; motivation
As components of the limbic system, the amygdala, nucleus accumbens (NAc), and prefrontal cortex (PFC) are involved in interdependent functional modalities that coordinate emotional and cognitive behavior. The amygdala is thought to mediate the processing and expression of emotional behavior (Davis, 1992; LeDoux, 1992; Gallagher and Holland, 1994), the PFC is implicated in cognitive flexibility and decision-making based on stimulus value and expected outcome (Damasio, 1994; GoldmanRakic, 1995; Robbins, 1996), and the NAc provides an interface where stimuli with affective and emotional value access the motor effector sites (Mogenson et al., 1980; Pennartz et al., 1994).

Multidisciplinary lines of investigation have described close anatomical and functional interactions between these structures. For example, reciprocal projections between subregions of the amygdala and PFC (Kelley et al., 1982; Groenewegen and Berendse, 1990) are implicated in cognitive regulation of emotional reactivity (Morgan and LeDoux, 1995; Goldstein et al., 1996; Schoenbaum et al., 1998; Baxter et al., 2000). Furthermore, glutamatergic afferents from PFC and the basolateral nucleus of the amygdala (BLA) to the shell region of the NAc are considered key pathways for expression of motor behavior that is driven by motivationally and emotionally relevant stimuli (Cador et al., 1989; Robbins et al., 1989). However, despite a great deal of

\footnotetext{
Received Aug. 21, 2000; revised Oct. 10, 2000; accepted Oct. 25, 2000.

This research was supported by National Institutes of Health Grants T32NS07224 (M.E.J.), K02MH01616 (B.M.), MH48404 (B.M.), MH44866 (B.M.), and the U.S. Veterans Administration Centers for Schizophrenia and Post-Traumatic Stress Disorder. We thank Barbara Adams and Karyn Groth for technical assistance.

Correspondence should be addressed to Bita Moghaddam, Department of Psychiatry, Yale University School of Medicine, 950 Campbell Avenue, VAMC 116A/2, West Haven, CT 06516. E-mail: bita.moghaddam@yale.edu.

Copyright (c) 2001 Society for Neuroscience 0270-6474/01/210676-06\$15.00/0
}

theoretical interest, experimental support for a triadic interaction, i.e., whether and how PFC provides an executive control over amygdala-driven response in the NAc, has been scarce (Simon et al., 1988; O’Donnell et al., 1999).

To explore the mechanisms by which PFC can modulate the dynamic interaction between BLA and NAc, we focused on dopamine release in the NAc of awake animals as a behaviorally relevant functional output measure. The dopaminergic innervation of the NAc is strongly implicated in the mediation of goaldirected behavior (Kelley et al., 1986; Taylor and Robbins, 1986; Le Moal and Simon, 1991; Salamone, 1991) and, more importantly, is considered necessary for expression of behavioral responding to BLA-dependent stimulus-reward associations (Cador et al., 1989; Everitt et al., 1991) and exploratory behavior (Yim and Mogenson, 1989). Experiments were performed in awake rats implanted with a stimulating electrode in the BLA and microdialysis probes in both the PFC and NAc for rapid measures of glutamate or dopamine efflux. The probe implanted in the PFC was also used for localized applications of compounds that manipulate glutamatergic neurotransmission during microstimulation of BLA. During microdialysis studies, stereotypical behavior was rated before, during, and after BLA stimulation.

\section{MATERIALS AND METHODS}

Animal preparation. All animal procedures were conducted in accordance with the National Institutes of Health Guide for the Care and Use of Laboratory Animals and were approved by the Yale University Animal Care and Use Committee. Male Sprague Dawley rats (280-350 gm) were anesthetized with halothane and placed in a stereotaxic frame with blunt ear bars. A small incision (7-10 mm) was made in the skin over the skull. The wound margin was infiltrated with lidocaine. Concentric microdialysis probes were implanted into the PFC [anteroposterior (AP), +3.2; lateral (L), 0.8; ventral (V), 5.3] and the NAc (AP, +1.4; L, 1.1; V, 8.3). 
A bipolar stainless steel electrode was implanted into the ipsilateral BLA (AP, -3.2; L, 5.0; V, 8.0). All coordinates are relative to bregma and according to the atlas of Paxinos and Watson (1982). Probes and an electrode were secured into place with dental acrylic and anchored by skull screws. Immediately after surgery, animals were placed in a clear polycarbonate cage $(44 \times 22 \times 42 \mathrm{~cm})$ with bedding, and the microdialysis probes were connected to a liquid swivel-balance arm assembly. This cage was placed in a quiet room with a $12 \mathrm{hr}$ light/dark cycle (lights on at 7:00 A.M., the same as the animal housing quarters). Animals had ad libitum access to food and water and were allowed to recover for at least $24 \mathrm{hr}$ before the start of microdialysis experiments and behavioral ratings, which were performed in the same cage/room environment.

Drugs and chemicals. LY354740 and LY293558 were gifts from Eli Lilly (Indianapolis, IN). Stock solutions $\left(10 \mathrm{~mm}\right.$ in $\left.\mathrm{H}_{2} 0\right)$ were prepared and kept frozen. Before use, stock solutions were diluted in the perfusion solution to a concentration of $1 \mu \mathrm{M}$ for LY354740 and $100 \mu \mathrm{M}$ for LY293558.

Microdialysis procedure. Concentric microdialysis probes with an outer diameter of $330 \mu \mathrm{m}$ and exposed tips of $3.0 \mathrm{~mm}$ (for PFC) and $2.0 \mathrm{~mm}$ (for NAc) were used. The perfusion solution contained (in mM): 145 $\mathrm{NaCl}, 2.7 \mathrm{KCl}, 1.0 \mathrm{MgCl}_{2}$, and $1.2 \mathrm{CaCl}_{2}$. Probes were perfused at a flow rate of $0.5 \mu \mathrm{l} / \mathrm{min}$ during the recovery period and $2.5 \mu \mathrm{l} / \mathrm{min}$ during the experiment. Dialysis samples were collected every 2 min for glutamate and every $10 \mathrm{~min}$ for dopamine. The amount of dopamine and glutamate in the samples was measured by HPLC as described elsewhere (Bagley and Moghaddam, 1997; Adams and Moghaddam, 1998).

Experimental procedures. Microdialysis and behavioral data were collected between 11:00 A.M. and 1:00 P.M. and after animals had acclimated to their new environment for $\sim 24 \mathrm{hr}$. Baseline samples were collected for $1 \mathrm{hr}$ before BLA stimulation. Separate groups of animals were used for dopamine and glutamate microdialysis studies, but data were collected from both PFC and NAc of the same animal. All data presented here are for BLA stimulation-naïve rats; therefore, separate groups of rats were used for the control group (for either glutamate or dopamine measures) and experiments involving intra-PFC perfusion of LY354740 or LY293558. Perfusion solution containing either drug was perfused starting $1 \mathrm{hr}$ before BLA stimulation.

Amygdala stimulation. Stimulation current was provided by a constant current source (Grass, Quincy, MA) driven by a pulse stimulator (Grass). The stimulation was delivered as a train of short bursts $(0.5 \mathrm{msec}$ monophasic pulses delivered at 200 pulses/sec for $20 \mathrm{msec}, 5$ pulses per burst) delivered at $1 \mathrm{sec}$ interburst intervals for a total duration of $10 \mathrm{~min}$.

Behavioral rating. During microdialysis studies, animals were rated for the 10 min periods before, during, and after BLA stimulation. Animals received a score of " 0 " for absence or " 1 " for presence of each of the following behaviors: ambulation, defined as movement about the cage; freezing, defined as an alert posture without movement; sniffing, defined as exploratory sniffing of the cage environment; mouth movement, defined as vacuous chewing motions of the mouth without food in the mouth; and jaw tremor, defined as a rapid quivering of the jaw, often resulting in an audible grinding noise. Note that because behaviors were scored if they persisted for at least $30 \mathrm{sec}$ during each $10 \mathrm{~min}$ epoch, seemingly contradictory behaviors (i.e., ambulation and freezing) could be observed in the same animal during the same epoch. Animals were scored only once every $10 \mathrm{~min}$ for a behavior even if it was exhibited repeatedly and for a duration longer than $30 \mathrm{sec}$. The scores are presented as the percentage of animals exhibiting defined behaviors for each experimental group. It should be emphasized that because a number of manipulations had to be performed immediately next to the animal (e.g., timed collection of microdialysis samples, turning the stimulator on/off, switching the perfusion solution to a drug-containing one, etc.), the rater was not blind to these manipulations.

Data analysis. All dialysis values given are expressed as percentages of baseline \pm SEM. The average concentration of three dialysis samples immediately before stimulation was defined as baseline. Within-group analysis of the main effect of stimulation was performed by using one-way ANOVA with time as the repeated measure. Between-group analysis was performed by using ANOVA with Bonferroni post hoc comparison of means. Behavioral data were analyzed by nonparametric Kruskal-Wallis ANOVA.

Histology. At the end of each experiment, animals were anesthetized with chloral hydrate. The location of the stimulating electrode was marked by passing a $5 \mathrm{~mA}$ current through the electrode for $30 \mathrm{sec}$. Animals were then perfused intracardially with saline followed by $10 \%$ buffered formalin. Brains were removed and stored in formalin. Fixed brains were cut at $250 \mu \mathrm{m}$ intervals, and sections were stained with cresyl violet. Probe placements were verified for all data presented this study and are demonstrated on Figure 1.

\section{RESULTS}

\section{Effects of BLA stimulation on glutamate efflux in PFC and NAC}

Stimulus parameters, glutamate response in PFC and NAc, and probe and electrode positions are summarized in Figure 1. Electrical stimulation of the BLA produced significant increases in extracellular glutamate in the PFC. This increase was immediate, peaked within 4 min of the beginning of the stimulation period, and persisted above baseline levels for $>20 \mathrm{~min}$. BLA stimulation also produced a significant increase in extracellular glutamate in the NAc. The magnitude of this increase was smaller than that observed in the PFC.

\section{Effects of BLA stimulation on dopamine efflux in PFC and NAC}

In the PFC, BLA stimulation produced a significant increase in extracellular dopamine levels (Fig. 2), which returned to baseline during the next $40 \mathrm{~min}$. In contrast to the PFC, there was no significant increase in dopamine efflux in the NAc during the period of BLA stimulation. A significant delayed increase was observed after the BLA stimulation was terminated. Dopamine levels in the NAc gradually returned to baseline during the next $50 \mathrm{~min}$.

\section{Effect of reduction of cortical glutamate activation during BLA stimulation on dopamine release in the NAc}

To test the hypothesis that the PFC regulates the output of NAc dopamine during BLA stimulation, we repeated the above experiments while blocking stimulus-induced glutamatergic activation in the PFC. We used two strategies to inhibit PFC glutamate neurotransmission. The first was to infuse into the PFC the selective AMPA/kainate receptor antagonist LY293558 to block postsynaptic glutamate receptor activation. Because this (commonly used) approach produces an indiscriminate blockade of glutamate neurotransmission, we also used another pharmacological strategy that produces presynaptic inhibition of evoked glutamate release (Lovinger and McCool, 1995; Battaglia et al., 1997) by stimulating group II metabotropic glutamate receptors (mGluR2/3). Because this approach has been primarily verified in vitro, we tested its effectiveness by applying the mGluR2/3 agonist LY354740 $(1 \mu \mathrm{M})$ to the PFC during BLA stimulation. As demonstrated in Figure 3, application of this compound effectively blocked stimulus-induced glutamate efflux.

In the subsequent experiments, either the AMPA receptor antagonist (LY293558, $100 \mu \mathrm{M}$ ) or the mGluR2/3 agonist (LY354740, $1 \mu \mathrm{M}$ ) was applied to the PFC while dopamine release was measured in both PFC and NAc (Fig. 4). In contrast to control animals in which no increase in NAc dopamine was observed during BLA stimulation, intra-PFC application of either compound resulted in an immediate increase in dopamine release in the NAc (Fig. 4a). In the case of LY293558, dopamine output remained elevated throughout the experiment. Local perfusion of LY354740 or LY293558 into the PFC had no significant effect on dopamine efflux in this region during BLA stimulation (Fig. 4b).

\section{Behavioral observations}

All animals were in a rest/sleep position before the start of BLA stimulation (data not shown). At the onset of the stimulation, animals became fully alert and exhibited several stereotypical 
a.

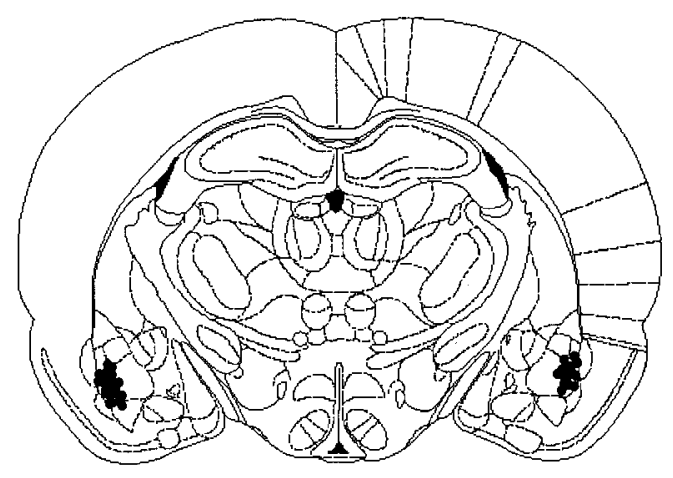

b.
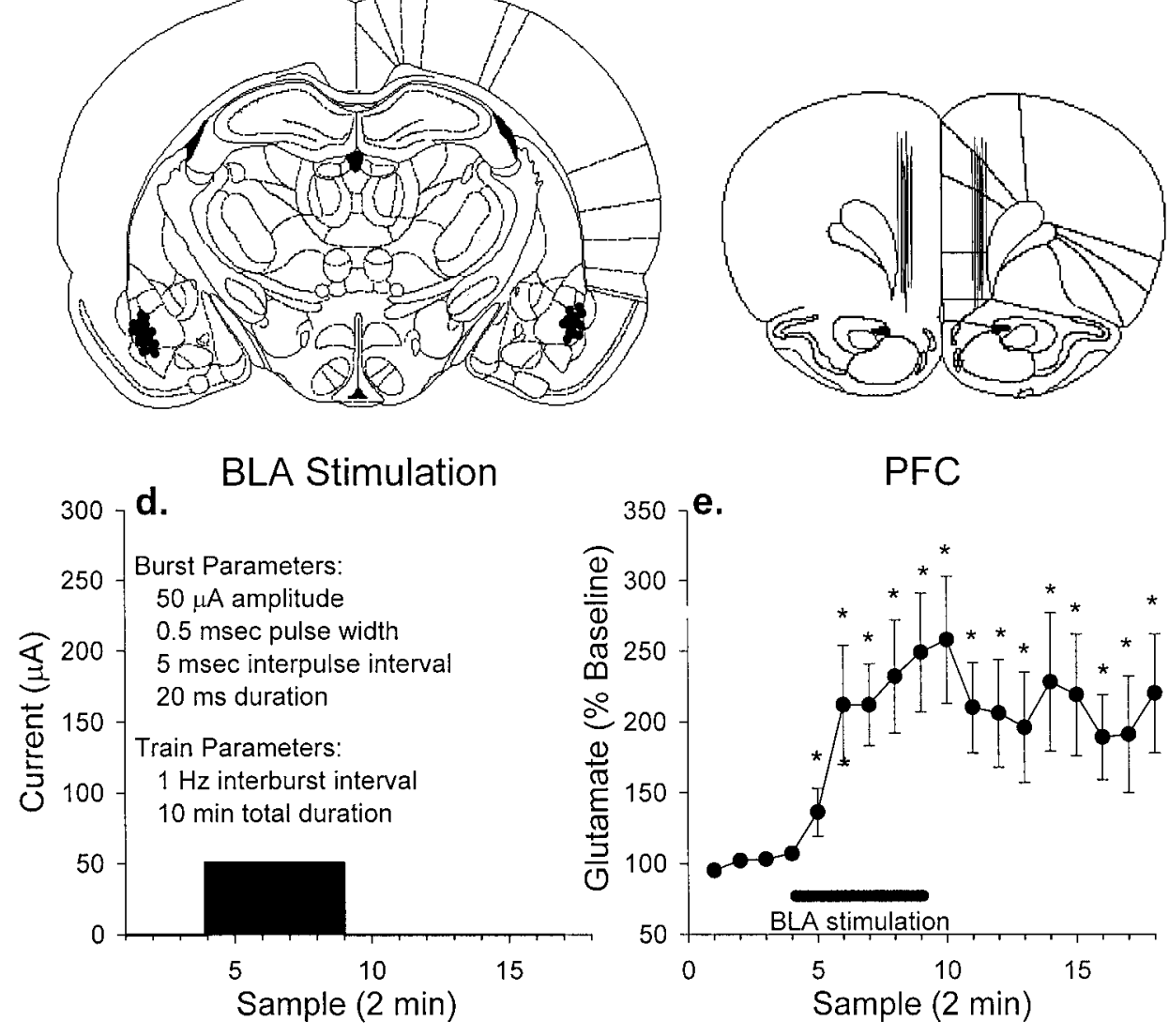

PFC

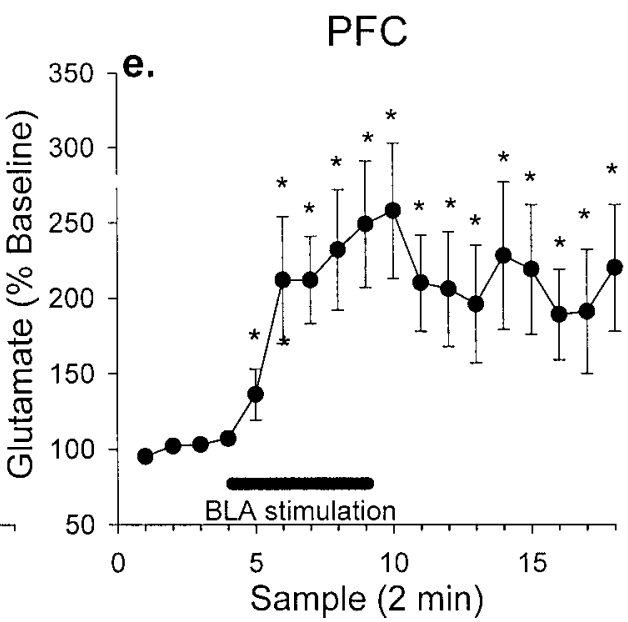

c.

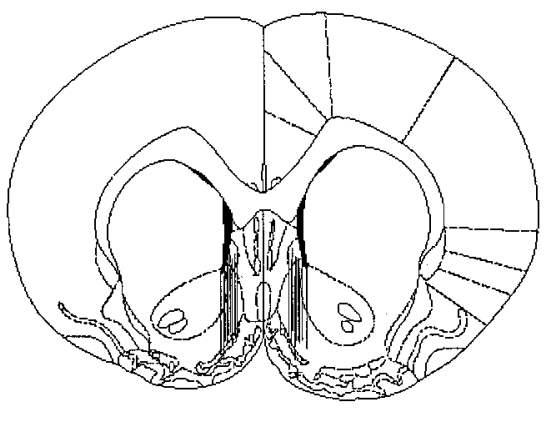

NAc

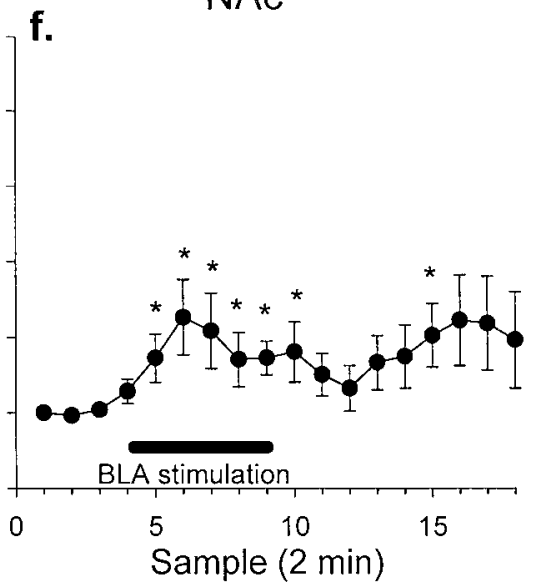

Figure 1. Placements of electrode and microdialysis probes, depiction of stimulus parameters, and glutamate efflux during BLA stimulation. Locations of electrode tips (-) and lines representing the active surface of microdialysis probes for all animals used in the study are illustrated. Electrode placement was ipsilateral to microdialysis probes in all experiments, but in different rats, placements were made randomly in either the left or right hemisphere. $a$, Electrode placements were confined to the basolateral amygdala complex. $b$, PFC probe placements were primarily in infralimbic/prelimbic regions of the medial PFC. $c$, NAc probe placements were along the border of the shell and medial core. $d$, BLA stimulation consisted of brief bursts of $50 \mu \mathrm{A}$ current pulses over a period of 10 min. $e$, BLA stimulation significantly increased glutamate efflux in the PFC $(F=4.53 ; p<0.01 ; n=15)$. $f$, BLA stimulation also increased glutamate efflux in the NAc $(F=1.69 ; p<0.05 ; n=10)$.

behaviors that are listed in Table 1. After the termination of the stimulus, the overall pattern of behavioral expression was different and included behaviors such as grooming and ambulation that were not observed during stimulation.

The behaviors displayed by animals with intra-PFC application of LY354740 or LY293558 differed from control animals both during and after BLA stimulation (Table 1). Aberrant behaviors observed during the stimulation, such as mouth movement and jaw tremor, were observed in a significantly higher percentage of these animals. In addition, they continued to express these behaviors after the termination of the stimulus, whereas normal poststimulus behaviors such as ambulation and grooming were observed in significantly fewer animals.

\section{DISCUSSION}

Microstimulation of the BLA at intensities that produced relatively mild behavioral activation produced an expected increase in glutamate efflux in the PFC and NAc. However, dopamine release increased only in the PFC, and not in the NAc, during the stimulation. An increase in NAc dopamine release was observed if stimulus-induced activation of glutamate neurotransmission in PFC was blocked, suggesting that PFC exerts an inhibitory control on amygdala-evoked activation of dopamine output in the NAc.
The PFC modulation of amygdala-NAc interaction is thought to provide an interface in which cognitive processes, such as on-line retention of internalized information, can influence amygdalamediated motor behavior (Mogenson et al., 1980; Damasio, 1994). Considering that activation of dopamine neurotransmission in the NAc elicits locomotor behavior (Kelley et al., 1986), this study suggests that a mechanism by which PFC can influence motor reactivity to amygdala-dependent stimuli is by downregulation of dopaminergic neurotransmission in the NAc.

\section{Glutamatergic and behavioral response to BLA stimulation}

In agreement with morphological studies describing glutamatergic projections from the BLA to PFC and NAc (Groenewegen and Berendse, 1990; Morgan and LeDoux, 1995), microstimulation of the BLA increased glutamate efflux in both regions. Although the densities of these innervations are comparable, BLA stimulation produced different patterns of response in PFC and NAc; in particular, the increase in extracellular glutamate was larger in magnitude and longer in duration in the PFC as compared with NAc. The smaller response in the NAc was surprising because, in addition to activation of amygdala-NAc projections, stimulation of PFC should also result in secondary 


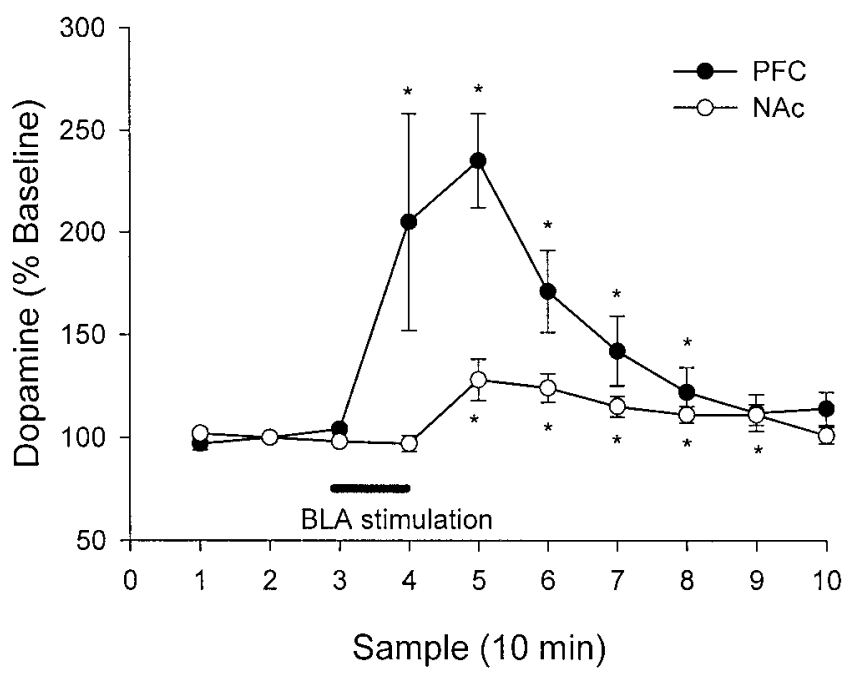

Figure 2. Dopamine release during BLA stimulation. BLA stimulation increased extracellular levels of dopamine in both the PFC $(F=6.09 ; p<$ $0.01 ; n=10)$ and the NAc $(F=4.65 ; p<0.01 ; n=10)$. However, post hoc analysis revealed that NAc dopamine levels did not increase during the period of BLA stimulation when compared with baseline. Asterisks indicate significant differences from baseline $(p<0.05)$.

activation of descending glutamatergic projections from PFC, a principal source of excitatory afferents to NAc (Sesack et al., 1989). Nonetheless, the observed pattern of glutamate increase suggests that BLA stimulation has a greater impact on activating glutamatergic neurotransmission in PFC than in NAc.

The behaviors exhibited by animals during the BLA stimulation are consistent with the involvement of this region in the expression of fear and anxiety. These behaviors (e.g., freezing, jaw tremor) were quite distinct from behaviors observed after the session of stimulation (e.g., grooming, ambulation) which generally are considered behaviors that contribute to adaptation to external environment. The sustained increase in PFC glutamate efflux may be relevant to the poststimulus behavioral activation. The observation that a normal pattern of poststimulus behavioral expression was not observed in animals in which the increase in glutamate release was blocked by the mGluR2/3 agonist, or when postsynaptic glutamate activation was blocked by an AMPA antagonist, is consistent with a role for PFC glutamate in sustaining the poststimulus behavioral activation.

\section{Dopamine response to BLA activation}

BLA stimulation produced a robust increase in dopamine efflux in the PFC. This increase was not blocked by inhibiting glutamate release in the PFC during the stimulation. Thus, it is likely that the activation of dopamine release results from increased neuronal activity in the ventral tegmental area (VTA) in which mesoprefrontal dopamine cell bodies are localized, as opposed to presynaptic regulation of dopamine by glutamate at the terminal (PFC) level. Although convincing evidence for direct projections from BLA to VTA is generally lacking in rodents, indirect projections through other structures may lead to activation of these neurons during BLA stimulation.

Release of dopamine in the NAc did not increase during BLA stimulation, although a delayed increase, starting after the cessation of stimulation, was observed. This latter increase is consistent with a delayed increase in accumbal dopamine reported after exposure to stress (Puglisi-Allegra et al., 1991) that has been

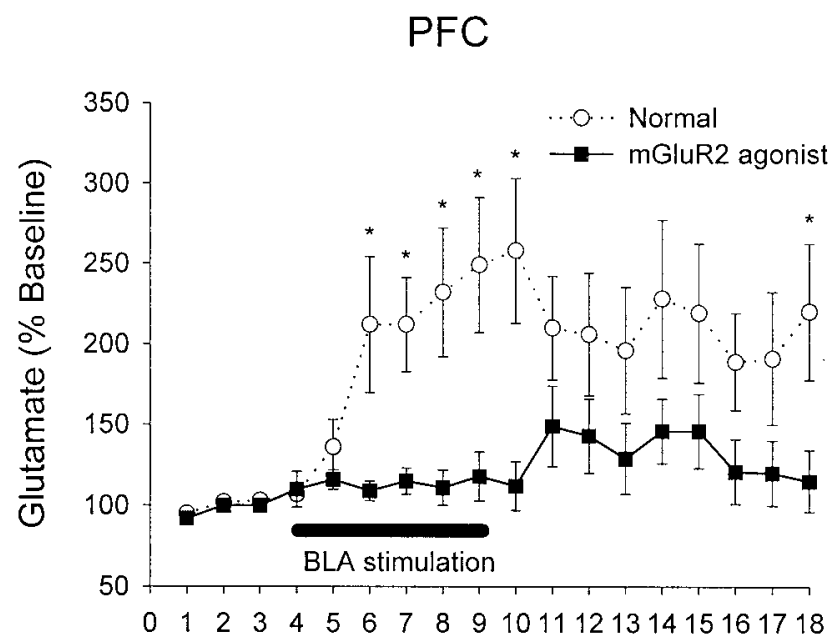

Sample ( 2 min)

Figure 3. Effect of intra-PFC perfusion of mGluR2/3 agonist LY354740 on glutamate efflux in the PFC during BLA stimulation. Reverse dialysis of LY354740 $(1 \mu \mathrm{M})$ reduced the glutamate efflux evoked by BLA stimulation (solid line; $n=7$ ) as compared with control animals (dotted line; $n=15$ ) (also depicted in Fig. 1). Two-way repeated-measures ANOVA revealed a significant difference between the groups $(F=12.04 ; p<0.01)$. Asterisks indicate individual samples that were significantly different between groups $(p<0.05)$. [Of note, we were able to detect LY354740 levels in our HPLC system that were used to detect glutamate, and therefore we were able to confirm that LY354740 did not diff use from the PFC to the NAc because, during the time that the compound was perfused into the PFC, it was not detected in the dialysate obtained from the probe implanted in the NAc (data not shown).]

attributed to the positive valence associated with termination of unpleasant stimuli.

The lack of a stimulus-locked increase in dopamine in the NAc was reversed after local perfusion of the mGluR2/3 agonist LY354740 and the AMPA/kainate antagonist LY293558 into the PFC. Assuming that LY354740 presynaptically inhibits stimulated glutamate release, an assumption supported by data presented in Figure 3, this finding indicates that activation of glutamate release in the PFC inhibits dopamine release in the NAc during BLA stimulation. Blockade of AMPA receptors in the PFC, which reduces the postsynaptic effects of BLA-mediated glutamate activation, produced a similar response, providing additional support that an increase in glutamate neurotransmission in the PFC downregulates dopamine release in NAc during BLA activation.

Through what mechanism might amygdala activation differentially regulate dopamine release in the PFC and NAc? Dopamine neurons localized in the VTA appear to receive target-specific input from afferent regions (Carr and Sesack, 2000). In particular, afferents from the PFC make synaptic contact with dopamine, and not GABA, VTA neurons that project back to the PFC. In contrast, dopamine neurons that project to the NAc do not receive direct $\mathrm{PFC}$ input, although GABA interneurons and GABAergic projections to the NAc make synaptic contact with cortical afferents (Carr and Sesack, 2000). This pattern of innervation suggests that activation of PFC efferents can (indirectly) inhibit VTA dopamine neurons that project to NAc but stimulate dopamine neurons that project to the PFC. This bimodal mechanism is consistent with the opposite pattern of dopamine activity in PFC and NAc in behaviorally relevant contexts (Piazza et al., 1991) and with neurochemical studies, indicating that dopamine projections to the PFC are under a tonic excitatory control of 
Figure 4. Effects of local perfusion into PFC of mGluR2/3 agonist LY354740 (1 $\mu \mathrm{M})$ and AMPA antagonist LY293558 $(100 \mu \mathrm{M})$ on dopamine efflux in the NAc and PFC during BLA stimulation. Drug perfusion began at least $1 \mathrm{hr}$ before BLA stimulation. $a$, BLA stimulation increased NAc dopamine efflux with either the mGluR2/3 agonist in the PFC (solid line, $\square)(F=3.72 ; p<0.01 ; n=9)$ or the AMPA antagonist in the PFC (solid line, 口) $(F=4.26 ; p<0.01 ; n=6)$. This increase was in contrast to the lack of immediate dopamine increase during BLA stimulation in control rats (dotted line) (also depicted in Fig. 2.). Asterisks indicate significant differences between the control and the other two groups $(p<$ $0.05)$. $b$, In the PFC, dopamine increase was evoked by BLA stimulation in the presence of either the mGluR2/3 agonist (solid line, $\square)(F=11.9 ; p<0.01 ; n=8)$ or the AMPA antagonist (solid line, $\mathbf{\square})$ $(F=2.22 ; p<0.05 ; n=8)$. There were no significant differences between these groups and the control group.

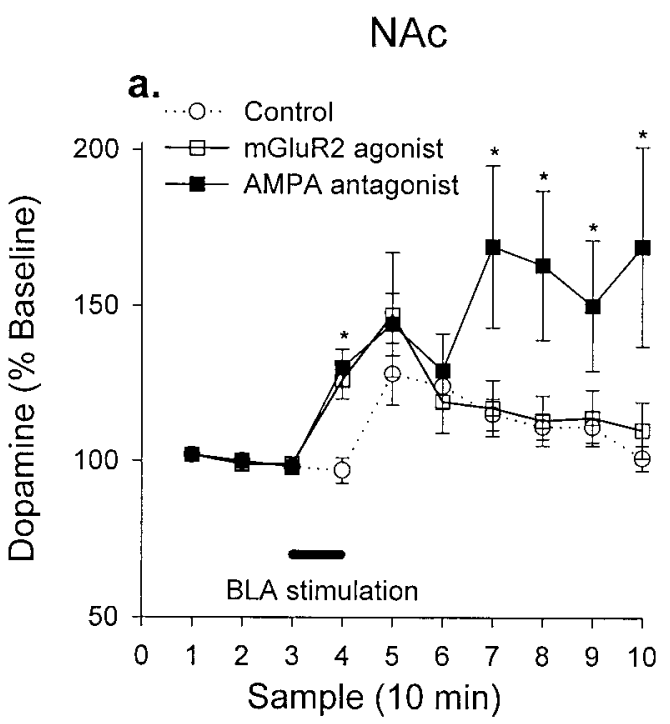

Table 1. Behaviors observed during microdialysis sample collection in the $\mathbf{1 0}$ min periods during and after BLA stimulation

\begin{tabular}{|c|c|c|c|c|c|c|}
\hline \multirow[b]{2}{*}{ Behaviors } & \multicolumn{2}{|l|}{ Controls } & \multicolumn{2}{|c|}{ LY354740 } & \multicolumn{2}{|c|}{ LY293558 } \\
\hline & During & After & During & After & During & After \\
\hline Ambulation & & 63 & 0 & $13^{*}$ & 0 & 33 \\
\hline Grooming & 0 & 59 & 0 & 25 & 0 & $11^{*}$ \\
\hline Freezing & 96 & 56 & 94 & 81 & 100 & 56 \\
\hline Sniffing & 48 & 63 & $6^{*}$ & 50 & 22 & 56 \\
\hline Mouth movement & 63 & 26 & 81 & $69^{*}$ & $100 *$ & $67 *$ \\
\hline Jaw tremor & 48 & 33 & 31 & 38 & $78^{*}$ & $89^{*}$ \\
\hline
\end{tabular}

Data are presented as percentages of animals that exhibited selected behaviors within each group. The control group $(n=$ 27) had normal Ringer's perfused into the microdialysis probe implanted in the PFC. In the other two groups, either the mGluR2 agonist LY354740 $(n=16)$ or the AMPA/kainate antagonist LY293558 $(n=9)$ was added to the Ringer's solution that was perfused into the PFC. All animals were at rest for the 10 min period before BLA stimulation (data not shown). Asterisks denote significant differences $(p<0.05)$ compared with the control group.

glutamate, whereas mesoaccumbens dopamine neurons are tonically inhibited by glutamate (Takahata and Moghaddam, 2000). Based on this mechanism, the BLA stimulus-induced increase in PFC glutamate neurotransmission observed in the present study, which should result in activation of PFC efferents to the VTA, would be expected to inhibit VTA dopamine neurons that project to the NAc. Blockade of PFC glutamate activation during BLA stimulation would, therefore, attenuate the inhibitory influence of PFC on mesoaccumbens neurons and result in stimulus-locked activation of NAc dopamine. This was, in fact, our observation. Thus, although stimulation of amygdala may activate the glutamatergic input to all VTA dopamine neurons (via direct or indirect pathways), concomitant activation of glutamate neurotransmission in the PFC selectively counteracts this excitatory effect on dopamine neurons that project to the NAc.

In contrast to the present findings, the general notion has been that PFC exerts an excitatory control over dopamine release in the NAc. This mechanism has been supported by studies showing that electrical stimulation of PFC (Taber and Fibiger, 1995; You et al., 1998) or blockade of GABA inhibitory tone by intra-PFC application of bicuculline (Karreman and Moghaddam, 1996) produces an increase in dopamine release in the NAc. The electrical stimulation frequencies $(60-400 \mathrm{~Hz})$ used in these studies may be considered nonphysiological because single unit firing rates of PFC neurons in rats during performance of PFCdependent cognitive tasks do not exceed $10 \mathrm{~Hz}$ (Jung et al., 1998). Thus, application of bicuculline or these high-intensity stimulations may increase NAc dopamine release because they result in glutamate spillover or other extrasynaptic mechanisms that may produce indiscriminate activation of dopamine neurons. These findings suggest that depending on the intensity of stimulation, or other conditions that influence basal cortical activity, the PFC may exert a bi-phasic influence on NAc dopamine release (Carlsson et al., 1999).

A previous study using in vivo voltammetry in anesthetized animals reported that NAc dopamine release increases during BLA activation (Floresco et al., 1998). Although this study may appear to contradict the present results, considering that cortical activity is generally depressed under anesthesia, the voltammetry study actually supports our conclusion that if PFC is taken offline, activation of the BLA readily increases dopamine efflux in the NAc. Indirect support for an amygdala-mediated inhibition of NAc dopamine in awake animals can also be found in other studies (Simon et al., 1988; Rada and Hernandez, 1990; Louilot 
and Besson, 2000), including the classic study by Yim and Mogenson (1989) showing that motor retardation induced by chemical stimulation of amygdala is reversed by intra-accumbal application of dopamine.

\section{Conclusions}

Cortical modulation of information transfer from amygdala to NAc is considered essential for translation of cognitive and emotive processes into appropriate motor responding. PFC control has been considered to be primarily, if not exclusively, at the postsynaptic level, i.e., by direct activation of NAc output neurons (Pennartz et al., 1994; O'Donnell et al., 1999). This study suggests that inhibiting the output of mesoaccumbens dopamine neurons is a mechanism by which PFC may work in concert with the amygdala to produce prolonged modulation of neurotransmission in the NAc. This is significant in light of the proposed role of NAc dopamine in the expression of goal-directed behavior (Mogenson et al., 1980; Le Moal and Simon, 1991; Salamone, 1991) and amygdala-mediated stimulus-reward association (Cador et al., 1989). Thus, cognitive processes specialized to the PFC, such as on-line retention of internalized information (Goldman-Rakic, 1995), may influence behavioral responding to emotional input from the amygdala through a PFC-mediated inhibition of NAc dopamine. Our behavioral observations further suggest that the absence of this PFC control may result in aberrant behavioral responding during amygdala activation and response perseveration after stimulus termination.

\section{REFERENCES}

Adams B, Moghaddam B (1998) Corticolimbic dopamine neurotransmission is temporally dissociated from the cognitive and locomotor effects of phencyclidine. J Neurosci 18:5545-5554.

Bagley J, Moghaddam B (1997) Temporal dynamics of glutamate efflux in the prefrontal cortex and in the hippocampus following repeated stress: effects of pretreatment with saline or diazepam. Neuroscience 77:65-73.

Battaglia G, Monn JA, Schoepp DD (1997) In vivo inhibition of veratridine-evoked release of striatal excitatory amino acids by the group II metabotropic glutamate receptor agonist LY354740 in rats. Neurosci Lett 229:161-164.

Baxter M, Parker A, Lindner C, Izquierdo A, Murray E (2000) Control of response selection by reinforcer value requires interaction of amygdala and orbital prefrontal cortex. J Neurosci 20:4311-4319.

Cador M, Robbins T, Everitt B (1989) Involvement of the amygdala in stimulus-reward associations: interaction with the ventral striatum. Neuroscience 30:77-86.

Carlsson A, Waters N, Carlsson M (1999) Neurotransmitter interactions in schizophrenia: therapeutic implications. Biol Psychiatry 46:1388-1395.

Carr D, Sesack S (2000) Projections from the rat prefrontal cortex to the ventral tegmental area: target specificity in the synaptic associations with mesoaccumbens and mesocortical neurons. J Neurosci 20:3864-3873.

Damasio A (1994) Descartes' error: emotion, reason, and the human brain. New York: Grosset/Putnam.

Davis M (1992) The role of the amygdala in conditioned fear. In: The amygdala: neurological aspects of emotion, memory, and mental dysfunction (Aggleton J, ed), pp 255-306. New York: Wiley.

Everitt B, Morris K, O'Brien A, Burns L, Robbins T (1991) The basolateral amygdala-ventral striatal system and conditioned place preference: further evidence of limbic-striatal interactions underlying reward-related processes. Neuroscience 42:1-18.

Floresco S, Yang C, Phillips A, Blaha C (1998) Basolateral amygdala stimulation evokes glutamate receptor-dependent dopamine efflux in the nucleus accumbens of the anesthetized rat. Eur $\mathbf{J}$ Neurosci 10:1241-1251.

Gallagher M, Holland P (1994) The amygdala complex: multiple roles in associative learning and attention. Proc Natl Acad Sci USA 91:1177111776.

Goldman-Rakic P (1995) Cellular basis of working memory. Neuron 14:477-485.

Goldstein L, Rasmusson A, Bunney B, Roth R (1996) Role of the amygdala in the coordination of the behavioral, neuroendocrine, and prefrontal cortical monoamine responses to psychological stress in the rat. J Neurosci 16:4787-4798.

Groenewegen H, Berendse H (1990) Connections of the subthalamic nucleus with ventral striatopallidal parts of the basal ganglia in the rat. J Comp Neurol 294:607-622.

Jung M, Qin Y, McNaughton B, Barnes C (1998) Firing characteristics of deep layer neurons in prefrontal cortex in rats performing spatial working memory tasks. Cereb Cortex 8:437-450.

Karreman M, Moghaddam B (1996) The prefrontal cortex regulates the basal release of dopamine in the limbic striatum: an effect mediated by ventral tegmental area. J Neurosci 66:589-598.

Kelley A, Domesick V, Nauta W (1982) The amygdalostriatal projection in the rat: an anatomical study by anterograde and retrograde tracing methods. Neuroscience 7:615-630.

Kelley A, Winnock M, Stinus L (1986) Amphetamine, apomorphime, and investigatory behavior in the rat: analysis of the structure and pattern of responses. Psychopharmacology (Berl) 88:66-74.

LeDoux J (1992) Brain mechanisms of emotion and emotional learning. Curr Opin Neurobiol 2:191-198.

Le Moal M, Simon H (1991) Mesocorticolimbic dopaminergic network: functional and regulatory roles. Physiol Rev 71:155-234.

Louilot A, Besson C (2000) Specificity of amygdalostriatal interactions in the involvement of mesencephalic dopaminergic neurons in affective perception. Neuroscience 96:73-82.

Lovinger DM, McCool BA (1995) Metabotropic glutamate receptormediated presynaptic depression at corticostriatal synapses involves mGluR2 or 3. J Neurophysiol 73:1076-1083.

Mogenson G, Jones D, Yim C (1980) From motivation to action: functional interface between the limbic system and the motor system. Prog Neurobiol 14:69-97.

Morgan M, LeDoux J (1995) Differential contribution of dorsal and ventral medial prefrontal cortex to the acquisition and extinction of conditioned fear in rats. Behav Neurosci 109:681-688.

O'Donnell P, Greene J, Pabello N, Lewis BL, Grace AA (1999) Modulation of cell firing in the nucleus accumbens. Ann NY Acad Sci 877:157-175.

Paxinos G, Watson C (1982) The rat brain in stereotaxic coordinates. New York: Academic.

Pennartz C, Groenewegen H, Lopez da Silva FH (1994) The nucleus accumbens as a complex of functionally distinct neuronal ensembles: an integration of behavioural, electrophysiological and anatomical data. Prog Neurobiol 42:719-761.

Piazza PV, Rouge-Pont F, Deminiere JM, Kharoubi M, Le Moal M, Simon H (1991) Dopaminergic activity is reduced in the prefrontal cortex and increased in the nucleus accumbens of rats predisposed to develop amphetamine self-administration. Brain Res 567:169-174.

Puglisi-Allegra S, Imperato A, Angelucci L, Cabib S (1991) Acute stress induces time-dependent responses in dopamine mesolimbic system. Brain Res 554:217-222

Rada P, Hernandez L (1990) Opposite changes of dopamine turnover in prefrontal cortex and nucleus accumbens after amygdaloid kindling. Neurosci Lett 117:144-148.

Robbins T (1996) Dissociating executive functions of the prefrontal cortex. Philos Trans R Soc Lond B Biol Sci 351:1463-1470.

Robbins T, Cador M, Taylor J, Everitt B (1989) Limbic-striatal interactions in reward-related processes. Neurosci Biobehav Rev 13:155-162.

Salamone J (1991) Behavioral pharmacology of dopamine systems: a new synthesis. In: The mesolimbic dopamine system: from motivation to action (Wilner P, Scheel-Kruger J, eds). New York: Wiley.

Schoenbaum G, Chiba A, Gallagher M (1998) Orbitofrontal cortex and basolateral amygdala encode expected outcomes during learning. Nat Neurosci 1:155-159.

Sesack S, Deutch A, Roth R, Bunney B (1989) Topographical organization of the efferent projections of the medial prefrontal cortex in the rat: an anterograde tract-tracing study with Phaseolus vulgaris leucoagglutinin. J Comp Neurol 290:213-242.

Simon H, Taghzouti K, Gozlan H, Studler JM, Louilot A, Herve D, Glowinski J, Tassin JP, Le Moal M (1988) Lesion of dopaminergic terminals in the amygdala produces enhanced locomotor response to D-amphetamine and opposite changes in dopaminergic activity in prefrontal cortex and nucleus accumbens. Brain Res 447:335-340.

Taber M, Fibiger H (1995) Electrical stimulation of the prefrontal cortex increases dopamine release in the nucleus accumbens of the rat: modulation by metabotropic glutamate receptors. J Neurosci 15:3896-3904.

Takahata R, Moghaddam B (2000) Target-specific glutamatergic regulation of dopamine neurons in the ventral tegmental area. J Neurochem 75:1775-1778.

Taylor J, Robbins T (1986) 6-Hydroxydopamine lesions of the nucleus accumbens, but not the caudate nucleus, attenuate responding with reward-related stimuli produced by intra-accumbens D-amphetamine. Psychopharmacology (Berl) 90:390-397.

Yim C, Mogenson G (1989) Low doses of accumbens dopamine modulate amygdala suppression of spontaneous exploratory activity in rats. Brain Res 477:202-210.

You Z, Tzschentke T, Brodin E, Wise R (1998) Electrical stimulation of the prefrontal cortex increases cholecystokinin, glutamate, and dopamine release in the nucleus accumbens: an in vitro microdialysis study in freely moving rats. J Neurosci 18:6492-6500. 